\author{
RESEARCH ARTICLE \\ 10.1029/2018JC014106 \\ Key Points: \\ - A Lagrangian model is developed for \\ changes in salinity and isotopic \\ composition of a moving water \\ column that undergoes net \\ evaporation \\ - The model is successfully applied to \\ four oceanic transects \\ - The observations provide reliable \\ estimates of the model's new \\ single-parameter - evaporation \\ length
}

Correspondence to:

N. Paldor,

nathan.paldor@mail.huji.ac.il

Citation:

Berman, H., Paldor, N., Churchill, J., \& Lazar, B. (2019). Constraining evaporation rates based on large-scale sea surface transects of salinity or isotopic compositions. Journal of Geophysical Research: Oceans, 124, 1322-1330. https://doi.org/10.1029/ 2018JC014106

Received 23 APR 2018 Accepted 23 JAN 2019 Accepted article online 26 JAN 2019 Published online 27 FEB 2019

C2019. American Geophysical Union. All Rights Reserved.

\section{Constraining Evaporation Rates Based on Large-Scale Sea Surface Transects of Salinity or Isotopic Compositions}

\author{
Hadar Berman $^{1}$ (D), Nathan Paldor ${ }^{1}$ (D) James Churchill ${ }^{2}$, and Boaz Lazar ${ }^{1}$ (D) \\ ${ }^{1}$ The Fredy and Nadine Herrmann Institute of Earth Sciences, Hebrew University of Jerusalem, Jerusalem, Israel, \\ ${ }^{2}$ Department of Physical Oceanography, Woods Hole Oceanographic Institution, Woods Hole, MA, USA
}

\begin{abstract}
A Lagrangian model is constructed for a surface column of initial height $h(0)$ that propagates at an average speed $u$ and is subject to excess (i.e., net) evaporation of $q \mathrm{~m} /$ year. It is shown that these parameters combine to form an evaporation length, $L=u h(0) / q$, which provides an estimate for the distance the column must travel before evaporating completely. While these changes in the surface water level due to evaporation are compensated by entrainment of water into the overall column, the changes in either near-surface salinity or isotopic compositions are retained and can be measured. Observations of surface salinity and isotopic compositions of $\delta^{18} O$ and $\delta D$ along 1,000- to 3,500-km long transects are used to estimate values of $L$ in the Red Sea, Mediterranean Sea, Indian Ocean, and Gulf Stream. The variations of salinity, $\delta^{18} O$ and $\delta D$ in all four basins are linear. As anticipated, the estimated value of $L$ is smallest in the slowly moving and arid Red Sea and is greatest in the fast-moving Gulf Stream.
\end{abstract}

Plain Language Summary a conceptual Lagrangian model is developed for constraining rates of evaporation in the ocean. The model is applied to routinely measured variables in the ocean's surface such as salinity and isotopic compositions. Our results show that all variables yield very similar estimates of the model's parameter, which bolsters the use of surface transects of salinity to constrain calculated values of parameters relevant to rates of evaporation.

\section{Introduction}

Evaporation of water to the atmosphere from the underlying sea is a dominant process driving the Earth's hydrologic cycle and the transfer of energy, in the form of latent heat, from the sea to the atmosphere. It is well known that the transfer of latent heat to the atmosphere due to evaporation and subsequent condensation provides the main energy source for the General Circulation of the Tropical Atmosphere (Manabe \& Smagorinsky, 1967). From an oceanographic point of view, basins characterized by intense positive net evaporation, such as the Mediterranean Sea (MS), have relatively high salinity values as compared with ocean basins, such as the Black Sea, in which evaporation is nearly balanced by the influx of fresh water (Talley et al., 2011).

The quantification of the net balance of evaporation minus precipitation and fresh water runoff in a basin is traditionally done by employing a box model and assuming that the total fluxes of water and salt in and out of the box vanish; that is, both the volume of water and the total mass of salt in the basin are in steady state. The conservation of water (i.e., volume) and salt in the basin yield the well-known Knudsen relation (Knauss, 1978; Talley et al., 2011). An identical approach is applied to unit volumes that surround grid-points in Ocean General Circulation Models (OGCMs). Both applications are Eulerian since they describe the conservation of mass (be it water or salt) or volume (of water) in a "box" that is fixed in space.

Quantification of this fundamental process of net evaporation is customarily done by applying empirical (called bulk) formulae that relate routinely observed meteorological variables, such as air temperature, sea surface temperature, wind, and relative humidity, to the amount of heat transferred to/from the atmosphere (see, e.g., Cayan, 1992; Knauss, 1978; Kondo, 1975).

In the present study, we employ the conservation of mass to a moving column that extends from the surface to some depth where net evaporation ceases to affect water properties such as salinity. The form of the continuity equation in the Lagrangian approach is identical to that employed in both OGCMs and box models but it incorporates the propagation of the column in the material time derivative. With this Lagrangian approach, transects of salinity or stable isotopes can be used to estimate a single parameter, the 


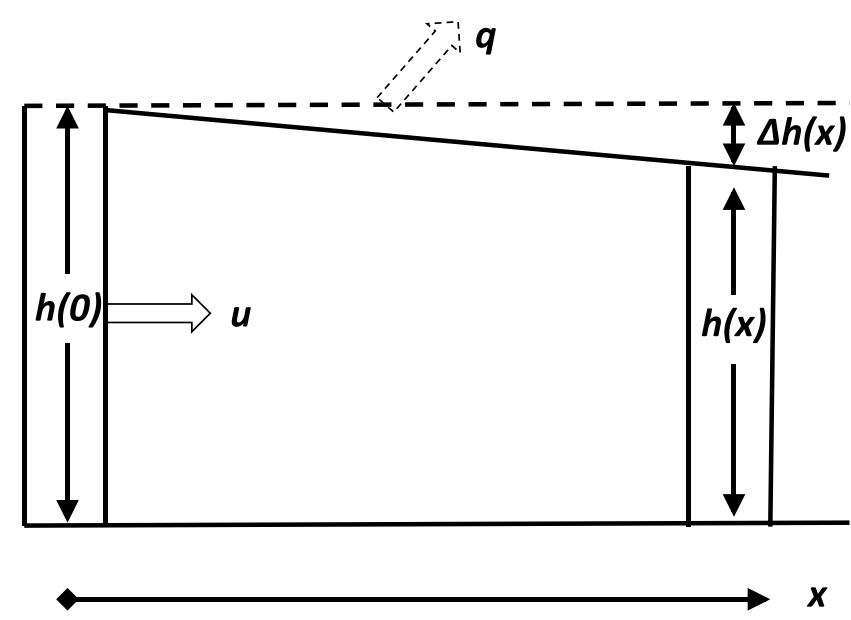

Figure 1. A sketch of the setup considered in this work: A column of water of thickness $h(0)$ is advected in the $x$ direction at mean speed $u$ while being subject to net evaporation rate $q$. At point $x$ along the transect the height of the column is $h(x)=h(0)-\Delta h(x)$. evaporation length, which can be used to characterize the strength of evaporation in a given ocean basin or current system.

The paper is arranged as follows. In section 2 we develop the Lagrangian conservation law for water, salt, and stable isotopes. The latter two serve as tracers that change along a transect due to the action of net evaporation. In section 3 we describe the various sources of data used in implementing our theory in different oceanic environments. In section 4 we apply the model results to the salinity and stable isotopes data. We show that estimates of the evaporation length derived in different basins are consistent with the differing intensity of evaporation in these basins, and that, in most cases, different tracers yield nearly equivalent estimates of the evaporation length. We end with a discussion of the utility of the model in section 5.

\section{Theory}

A sketch of the process under study is given in Figure 1, where a column of surface water of unit area and initial thickness $h(0)$ (e.g., the surface mixed layer) is advected at a mean speed $u$ over a distance $x$ while being subject to net evaporation (evaporation minus precipitation) that produces a change in depth at a rate of $q$ (e.g., in meters per year). This simple scenario typifies many oceanographic environments, such as flows in semi-enclosed basins (Ben-Sasson et al., 2009; POEM Group, 1992; Sofianos et al., 2002). More generally, it is applicable to any sufficiently long horizontal flow in which changes in surface salinity and isotopic composition due to evaporation are detectable by observations and regardless of the forcing/origin of the flow.

\subsection{Conservation of Water Volume}

The natural starting point for the study of changes in water properties induced by net evaporation, that is, evaporation minus precipitation, is the conservation of the mass of water in the moving column, that is, the Lagrangian perspective. For simplicity, we assume that the water density is constant to first order (e.g., $10^{3} \mathrm{~kg} / \mathrm{m}^{3}$ ) and ignore the second-order changes in the density that might take place due to changes in salinity. The rate at which mass of water evaporates from (i.e., leaves) the column of unit area depicted in Figure 1 due to the net evaporation of $q(\mathrm{~m} / \mathrm{s})$ is given by:

$$
\frac{\mathrm{d} h}{\mathrm{~d} t} \approx-\frac{\Delta h}{\Delta t}=-q,
$$

where $\Delta h=h(0)-h(x)>0$. Estimating $\Delta t$ in equation (1) by the advective time, $T_{\text {adv }} \equiv x / u$, then yields

$$
\Delta h(x)=\frac{q x}{u} .
$$

Although $u=0$ is formally a singular limit since $T_{\text {adv }}$ approaches infinity, it should be noted that in this case $x$-the distance traveled by the column-also vanishes so $T_{\text {adv }}$ is undefined (since it is the ratio of 0 divided by 0 ) but is not infinite, so in this limit $T_{\text {adv }}$ has to be defined by considerations other than the advective time $x / u$. Dividing equation (2) through by the initial thickness, $h(0)$, yields the nondimensional ratio between $\Delta h$ and $h(0)$ (denoted here by $g(x)$ ).

$$
g(x) \equiv \frac{\Delta h(x)}{h(0)}=\frac{q x}{u h(0)}=\frac{1}{L} x ; \quad \text { where } \quad L=\frac{u h(0)}{q} .
$$

The length $L=u h(0) / q$ is termed here evaporation length as it is the length that a column of initial thickness $h(0)$ has to travel at speed $u$ (so its travel time is $L / u$ ) in order for it to completely evaporate by the net evaporation $q$, that is, $\Delta h(x=L) \equiv q L / u=h(0)$ or $h(x)=0$. 
Our idealized Lagrangian model focuses on the primary effect of net evaporation and ignores other forcings, for example, wind, tides, and mixing that are considered second order. In realistic circumstances, additional oceanic processes (e.g., entrainment of water from below the mixed layer over which the surface column moves) compensate for the mass of water lost to the atmosphere so the changes in water mass, $\Delta h$, cannot be measured directly (i.e., as with satellite altimetry). The fact that such compensating processes that replenish the mass of water in the entire basin lost to the atmosphere exist, is readily appreciated by noting that on the large time scale considered here $\left(T_{\mathrm{adv}}\right.$ is much greater than the inertial period, i.e., about 1 day, see below) the sea surface slope has to be accompanied by a geostrophic velocity perpendicular to the slope itself, but such barotropic flows are not observed in the oceanic cases considered below. However, as shown below, the removal of mass of water from the column affects the salinity and isotopic composition in the column and these changes, which are proportional to $\Delta h$, are detectable by routine observations. The neglected forcings are expected to appear as noise in the signals of salinity or isotopic composition considered below.

Though the evaporation length, $L$, was developed in the context of the simple Lagrangian scenario depicted in Figure 1, we will shortly demonstrate that it is relevant to more complex and realistic scenarios as well. Such an application to observations should be attempted only subsequent to the development of explicit expressions that relate $L$ to changes in variables that are routinely observed in the ocean.

The function $g(x)$ can be interpreted by realizing that $h(0) / q$ (denoted here by $T_{\text {evap}}$ ) in equation (3) is the time it takes to evaporate the entire (mixed) layer of thickness $h(0)$. As was shown above, $x / u$ is the advective time $T_{\mathrm{adv}}$. It follows that in addition to the formal definition of $g(x)=\Delta h / h(0)$ (i.e., the fraction of $h(0)$ that was removed from the column), $g(x)$ is also the ratio of advective time to evaporation time:

$$
g(x) \equiv \frac{x}{L}=\frac{x q}{u h(0)}=\frac{\frac{x}{u}}{\frac{h(0)}{q}}=\frac{T_{\mathrm{adv}}}{T_{\mathrm{evap}}} .
$$

The straightforward considerations of conservation of mass/volume of water outlined here will be extended in the following subsections to the conservation of mass of salt and to the changes in isotopic composition in the column, both of which are affected by the removal of water molecules from the column during evaporation. These extensions will yield explicit expressions that relate changes in these variables to $g(x)$ or $L$.

\subsection{Conservation of Salt}

The simple scenario envisioned in Figure 1, which has surface water that is flowing while being subject to net evaporation, implies changes in the salinity of the water in the column. Typical evaporation rates are of order $2 \mathrm{~m} /$ year $\left(\approx 6.34 \cdot 10^{-8} \mathrm{~m} / \mathrm{s}\right)$. We assume that: (1) changes in density in the column associated with changes in salinity $(S)$ are second order only so they can be neglected and (2) that the mass of salt in the column is conserved, that is, $\frac{\mathrm{d}(S h)}{\mathrm{d} t}=0$, which leads to the following expression for salinity as a function of $x$ :

$$
S(x) h(x)=S(0) h(0) \Rightarrow S(0)=\left(1-\frac{\Delta h(x)}{h(0)}\right) S(x) .
$$

Here, $S(x)$ and $S(0)$ are the salinities at $x$ and at 0 , respectively. Applying the definition of $g(x)$ in equation (3), rearranging and using $g(x)<1$ then yields

$$
\frac{S(x)}{S(0)}=\frac{1}{1-g(x)}=1+g(x)+O\left(g^{2}\right) \Rightarrow \frac{S(x)-S(0)}{S(0)}=g(x)=\frac{1}{L} x+O\left(g^{2}\right),
$$

which implies that $S(x)$, increases linearly with $x$. Defining the increase in salinity along the transect, $\Delta S(x)=S(x)-S(0)>0$, equation (6) yields the following simple relation between the relative changes in salinity (i.e., salinity increase relative to the salinity at $x=0$ ) and $g(x)$ :

$$
\frac{\Delta S(x)}{S(0)}=g(x)=\frac{1}{L} x
$$

In the next subsection a similar relation is developed for changes in isotopic composition along the transect. 


\subsection{Changes in Stable Isotope Composition}

The oxygen isotopic composition of water undergoing evaporation while propagating in a semi-enclosed basin can be described using the Rayleigh equation (Appelo \& Postma, 2005; Craig et al., 1956; and equation 7 in Dansgaard, 1964):

$$
\delta^{18} O_{\text {water }}(x)=\delta^{18} O_{\text {water }}(0)+\varepsilon \ln (f)
$$

where $\delta^{18} O_{\text {water }}=\frac{R_{\text {water }}-R_{\text {standard }}}{R_{\text {standard }}} \cdot 1,000$ with $R_{\text {water }}$ and $R_{\text {standard }}$ the isotopic ratios ${ }^{18} \mathrm{O} /{ }^{16} \mathrm{O}$ (i.e., the rare to the abundant isotopes) of the water and of an internationally accepted standard, respectively. The remaining parameters in (8) are as follows: $f$ is the fraction of water remaining in the column at point $x$ (see Figure 1 ); $\varepsilon=1,000(\alpha-1)$ is the enrichment factor (in units of per mil) where $\alpha=\frac{R_{\text {vapor }}}{R_{\text {wate }}}$ is the isotope fractionation factor between vapor and water (where $R_{\mathrm{vapor}}$ and $R_{\mathrm{water}}$ are the isotopic ratios in the vapor and water phases, respectively). In this study $\alpha$ and $\varepsilon$ denote the fractionation and enrichment factors for evaporation only (i.e., condensation is excluded).

The definition of $f$ as the fraction of water remaining in the column after evaporation (see Figure 1) implies

$$
f=\frac{h(x)}{h(0)}=1-\frac{\Delta h(x)}{h(0)}
$$

Substituting this equation in Rayleigh equation, equation (8), yields

$$
\frac{\delta^{18} O_{\text {water }}(x)-\delta^{18} O_{\text {water }}(0)}{\varepsilon}=\ln \left(1-\frac{\Delta h(x)}{h(0)}\right)=-g(x)+O\left(g^{2}\right)=-\frac{1}{L} x .
$$

Relations similar to equations (8) and (10) determine the variation of hydrogen isotopes, $(D$ - the rare hydrogen isotope and ${ }^{1} \mathrm{H}$ - the abundant hydrogen isotope) along the transect. The only difference is that $\delta^{18} \mathrm{O}$ is replaced by $\delta D$ and the enrichment factor, $\varepsilon$ (as well as the fractionation factor, $\alpha$ ) are those of the hydrogen isotope.

Equations (6) and (10) relate changes in salinity and isotopic composition, respectively, in the advected column of Figure 1 to $g(x)$ In section 4 we will examine whether observed changes of salinity, $\delta D$ and $\delta^{18} O$ in the hypothetical column of Figure 1 vary linearly with $x$ in parts of the world ocean and whether they yield $g(x)$ slopes that are consistent with one another. The immediate candidates where this theory should be applied are semi-enclosed basins where evaporation is the primary driver of the advection. However, as we shall see, the theory is also applicable in circumstances where surface water is flowing because of other reasons (e.g., ocean size gyres) but is subject to excess evaporation during its flow.

In closing the theoretical part of this work, and prior to the application of this theory to oceanic observations, we note that for any particular oceanic transect of length $x_{\max }$ the function $g(x)$ in equation (3) yields a nondimensional number $\gamma=x_{\max } / L$ that quantifies the fraction of the original column that has evaporated while propagating over the entire transect of length $x_{\max }$. The expressions developed above in (6) and (10) are valid provided the neglected $O\left(g^{2}\right)$ terms are small, that is, $g\left(x_{\max }\right) \ll 1$ or $x_{\max } \ll L$. On the other hand, from a practical perspective, the application of these expressions to observations requires that $x_{\max }$ be long to ensure that $\Delta S(x)$ in (7) and changes in isotopic composition in (10) are significantly larger than the observational error. In the next section we describe the data that are used in section 4 to verify that the model is applicable to several oceanic locations in which $x_{\max } \geq 1,000 \mathrm{~km}$ and $L$ is at least 10 times larger so $g\left(x_{\max }\right) \ll 1$.

\section{Methods}

\subsection{Data}

As argued above, the Lagrangian model should be applied to observations along transects longer than $1,000 \mathrm{~km}$. Surface salinity transects of this length can be extracted from the Simple Ocean Data Assimilation (SODA, see details below) data set in any part of the world oceans. In contrast, stable isotope data are only available from dedicated cruises and when posted on accessible websites. One such cruise took place in the Red Sea (RS) in October-November 1998. The surface $\delta^{18} O$ and $\delta D$ (and salinity) data from this 


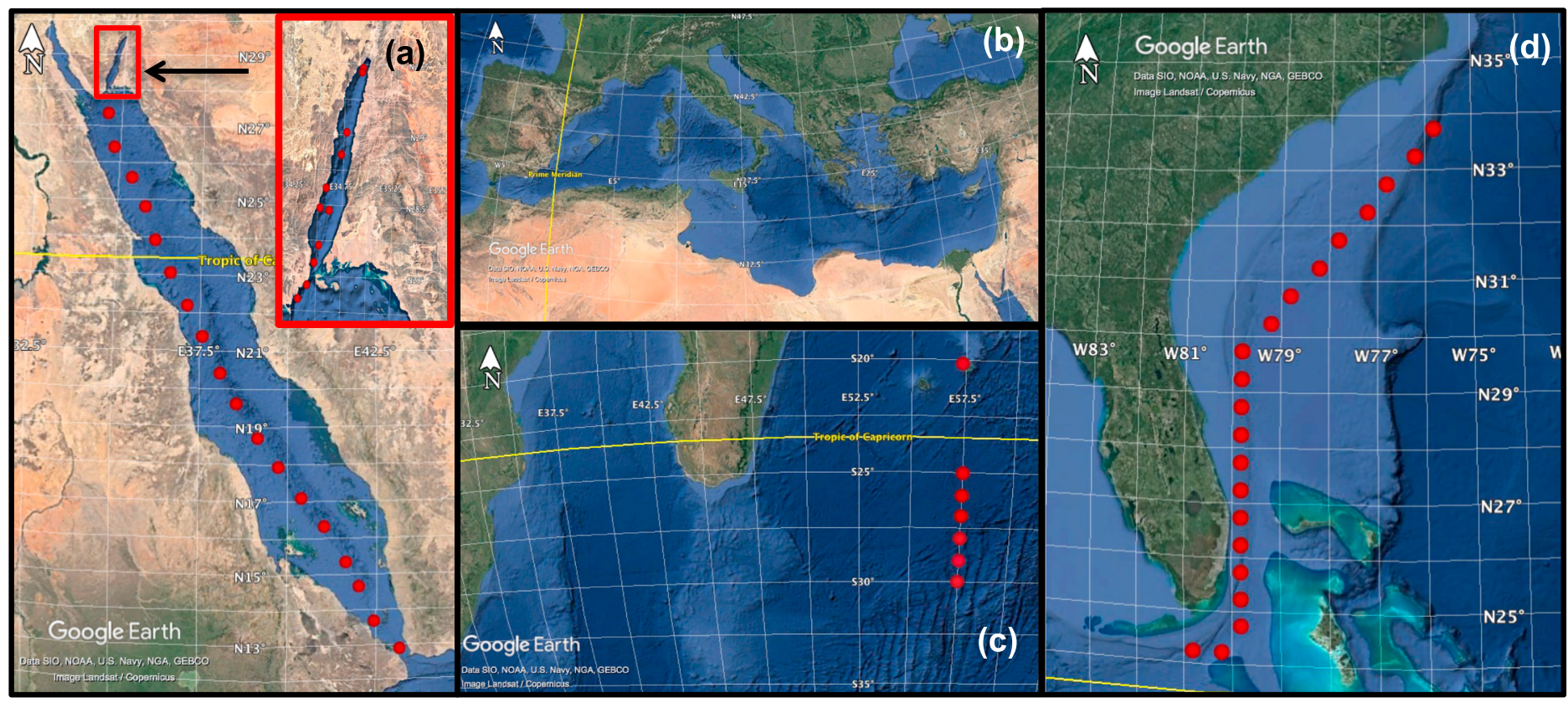

Figure 2. Maps of the four locations analyzed in this study. (a) Satellite image of the Red Sea with inset representing a zoom into the Gulf of Elat (Aqaba; adopted from Steiner et al., 2014). The red points represent sampling stations in the Red Sea and Gulf of Elat (Aqaba) where salinity, $\delta^{18} O$ and $\delta D$ were measured. (b) A satellite image of the Mediterranean Sea. (c) Satellite image of the Indian Ocean where surface salinity and $\delta^{18} O$ values at the stations marked by red dots were taken from Srivastava et al. (2007). (d) The Gulf Stream salinity transect is defined from Simple Ocean Data Assimilation surface velocities. Grid points where the speed exceeds $0.4 \mathrm{~m} / \mathrm{s}$ were averaged in the cross-stream direction to derive a single salinity value along the transect.

cruise were made available by Steiner et al. (2014). The second cruise, described by Srivastava et al. (2007), took place in the southwest Indian Ocean (IO, hereafter) in January-March 2006. The surface $\delta^{18} O$ (and salinity) data collected aboard this cruise can be accessed at https://www.nodc.noaa.gov. The data from these two areas were supplemented by salinity data from the MS, which, like the RS is a semi-enclosed evaporative basin, and the Gulf Stream (GS), where the surface speed is very high and $L$ should be extremely large according to (3). The four locations are shown in Figure 2, and the details of the data from these locations as well as the expected errors are described in the remainder of this section.

\subsubsection{SODA}

SODA data are based on reanalysis of available observations, supplemented by simulations by an OGCM (the Geophysical Fluid Dynamics Laboratory's Modular Ocean Model version 5). Carton et al. (2018) detail the sources of observations, the OGCM and the algorithm used for assimilating observations into the model. We employed SODA version 3.7.2 surface salinity data, which have a spatial and temporal resolution of $0.5^{\circ} \times 0.5^{\circ}$ and 1 month, respectively and can be downloaded from http://www.atmos.umd.edu/ ocean/. SODA data were recently applied by Ali et al. (2018) in a study of vertical and horizontal mass and heat fluxes over the central RS.

Six-year (2005-2010) averages of the SODA salinities at each grid point were used to determine salinities along the transects in our four target regions as detailed below.

Red Sea and Mediterranean Sea. Water enters these semi-enclosed basins through narrow straits (Bab-elMandeb and Gibraltar, respectively) and undergoes intense net evaporation along its route to the end points of basins (Elat/Aqaba and Israel/Lebanon, respectively, see Figures 2a and 2b) where the salinities are maximal. The RS transect shown in Figure 2a follows the stations occupied during the cruise of Steiner et al. (2014), while the SODA data extends to the tip of the Sinai Peninsula. In both the RS and MS salinity transects were formed by spatially averaging the temporally averaged SODA surface salinities across the mean surface flow, that is, zonally in the RS and meridionally in the MS.

Indian Ocean. The IO transect was selected along $58^{\circ} \mathrm{E}$ between $20^{\circ} \mathrm{S}$ and $30^{\circ} \mathrm{S}$ (see Figure $2 \mathrm{c}$ ) where the water flows poleward as part of the subtropical gyre of the southern hemisphere IO. The $58^{\circ} \mathrm{E}$ longitude of the SODA salinity transect was chosen to match the longtitude of the $\delta^{18} O$ data of Srivastava et al. (2007). 
Table 1

The values of Evaporation Length, L, Estimated From All Transects

\begin{tabular}{lccccc}
\hline & $L$ from SODA data $\left(10^{6} \mathrm{~m}\right)$ & & \multicolumn{2}{c}{$L$ from cruise data $\left(10^{6} \mathrm{~m}\right)$} \\
\cline { 2 - 2 } \cline { 5 - 6 } Location & Salinity & & Salinity & $\delta^{18} O$ & $\delta D$ \\
\hline RS & $26 \pm 1$ & & $22 \pm 1$ & $20 \pm 2$ & $28 \pm 3$ \\
MS & $87 \pm 5$ & & - & - & - \\
IO & $84 \pm 2$ & & $74 \pm 13$ & $35 \pm 5$ & - \\
GS & $500 \pm 40$ & & - & - & - \\
\hline
\end{tabular}

Gulf Stream. The definition of the GS used here is the stretch of ocean along the East Coast of the United States between $24^{\circ} \mathrm{N}-34^{\circ} \mathrm{N}$ and $76^{\circ} \mathrm{W}-81^{\circ} \mathrm{W}$ (between the Gulf of Mexico and Cape Hatteras, aka the Florida Current). SODA surface velocity data were used in the determination of the GS as the region where the velocity exceeds $0.4 \mathrm{~m} / \mathrm{s}$ (see Figure 2d). North of Cape Hatteras the meanders of the GS are too large for the salinity signal to be confidently identified in the 6-year averages. In the southern and northern segments (where the flow of the GS has a strong zonal component) the SODA values were averaged meridionally to yield a single value in the along-stream direction while in the central segment (where the GS flows northward) the SODA values were averaged zonally.

Errors. The error bars of SODA salinity data were calculated from the standard deviation of the spatial averages (RS, MS, and GS) and in the IO, where the transect is along the single longitude of $58^{\circ} \mathrm{E}$, the error bars were calculated from the temporal averages.

\subsubsection{Cruise Data}

Cruise data were used since it is the only source of stable isotopes data. For completeness, salinity data from these cruises were also analyzed.

Red Sea. Surface seawater samples were collected every $\sim 100 \mathrm{~km}$ along the RS (including the Gulf of Elat/Aqaba) using a specially designed water sampler. Each water sample yielded values of salinity, $\delta^{18} O$ and $\delta D$. Detailed information on the sampling of salinity and isotopic composition and the subsequent handling of these data can be found in Steiner et al. (2014).

Indian Ocean. The data used here are the salinity and $\delta^{18} \mathrm{O}$ between $20^{\circ} \mathrm{S}$ and $30^{\circ} \mathrm{S}$ along $58^{\circ} \mathrm{E}$ described in Srivastava et al. (2007) that are accessible over the web in the World Ocean Atlas (https://www.nodc.noaa. gov). According to Srivastava et al. (2007) the water in these latitudes are the Subtropic Mode Waters.

Errors. No information is available for the error bars in the salinity or stable isotope data collected in the two cruises.

\subsection{Linear Regression}

The observed variations of salinity and isotopic compositions along the four transects described in 3.1 were approximated by linear least squares regression functions that are related to the $g(x)$ function developed in section 2. According to equation (6), the intercept of $S(x)$ equals $S(0)$ and its slope equals $S(0) / L$. Thus, the value of $L$ in any salinity transect (from either SODA or cruise transects) is simply the intercept-to-slope ratio so the relative error in $L$ equals the sum of the relative errors in the intercept and slope of the linear regression line of $S(x)$.

The stable isotope data required that the value of $\varepsilon$ in equation (10) be determined for both $\delta^{18} O$ and $\delta D$ in order to determine the value of $L$. The slopes of these linear regression lines equal $-\varepsilon / L$ (note that for evaporation $\alpha<1$ which implies $\varepsilon<0$ so the isotopic composition increases with $x$ as does the salinity). For both $\delta^{18} O$ and $\delta D$ the relative errors in the values of $L$ are the sums of the errors in $\varepsilon$ and the relative errors in the slopes of the regression lines. For $\delta^{18} O$ during seawater evaporation, the values given in Majoube (1971) at temperature of $28^{\circ} \mathrm{C}$ (relevant to the RS and IO transects) are $\alpha_{18} \mathrm{O}^{16} \mathrm{O}=0.9909$, that is, $\varepsilon_{18} \mathrm{O} /{ }^{16} \mathrm{O}=-9.1 \%$. For $\delta D$ the values of Majoube (1971; quoted in Tables 1-4 in Clark \& Fritz, 1997) at temperature of $28{ }^{\circ} \mathrm{C}$ are $\alpha_{D / H}=0.9291$, that is, $\varepsilon_{D / H}=-70.9 \%$. Two sources of uncertainty in the calculated values of $\varepsilon_{18} O /{ }^{16} O$ were examined. First, we calculated it at slightly different temperature $\left( \pm 1.5^{\circ} \mathrm{C}\right)$ and second, we compared the above values with those given in Dansgaard (1964; section 1.2) based on slightly different values of the

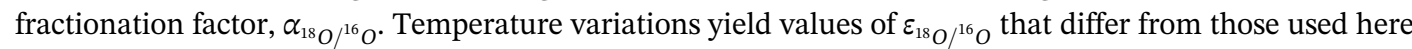
by about $1 \%$ and the Dansgaard's (1964) expressions for $\alpha^{18} \mathrm{O} /{ }^{16} \mathrm{O}$ yield values of $\varepsilon_{18} \mathrm{O} /{ }^{16} \mathrm{O}$ that differ by about $5 \%$. The combined effect of the two errors is smaller than the inherent error in the values of $L$ that vary between $10 \%$ and 15\% in both the RS and the IO (see also results in Table 1). Accordingly, the results reported below were calculated using the expressions given in Majoube (1971) and the reported error is that of the slope. 

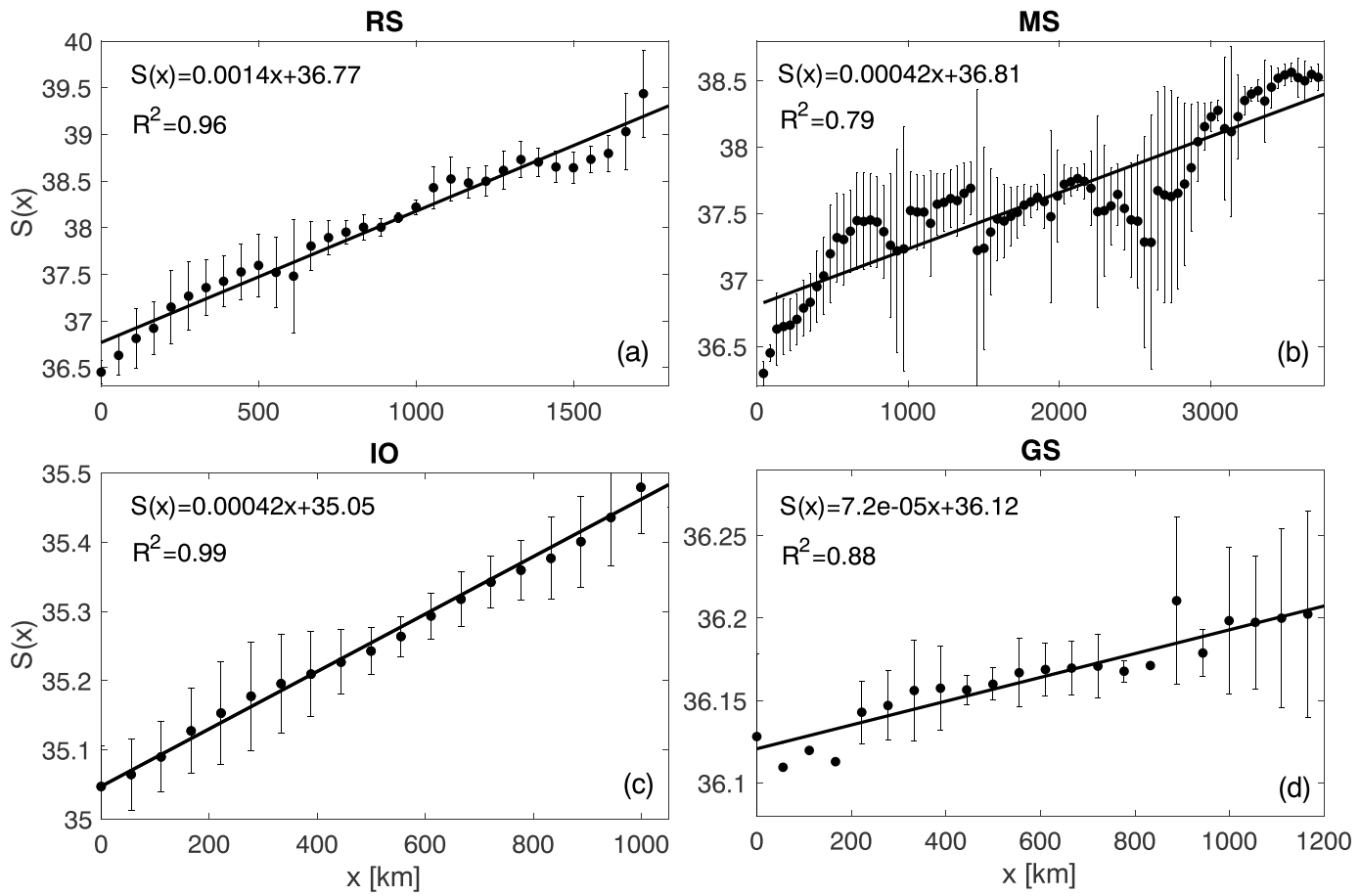

Figure 3. The variation of $S(x)$ along the four Simple Ocean Data Assimilation salinity transects listed in section 3.1.1. RS $=$ Red Sea; MS = Mediterranean Sea; IO = Indian Ocean; GS = Gulf Stream.

\section{Results}

The variations of $S(x)$ calculated from the four SODA salinity transects described in 3.1.1 are shown in Figure 3. The observed variations and the high $R^{2}$ values of the match between the data and the calculated linear regression lines clearly validate the prediction of the Lagrangian model, equation (6), that the variations are linear. The error bars of the GS transect increase significantly near the northern end at Cape Hatteras due to the increase of the meanders' amplitude there. The MS transect is highly variable and has the lowest $R^{2}$ value of 0.79 , which implies high inhomogeneity. The reason can be attributed to the width

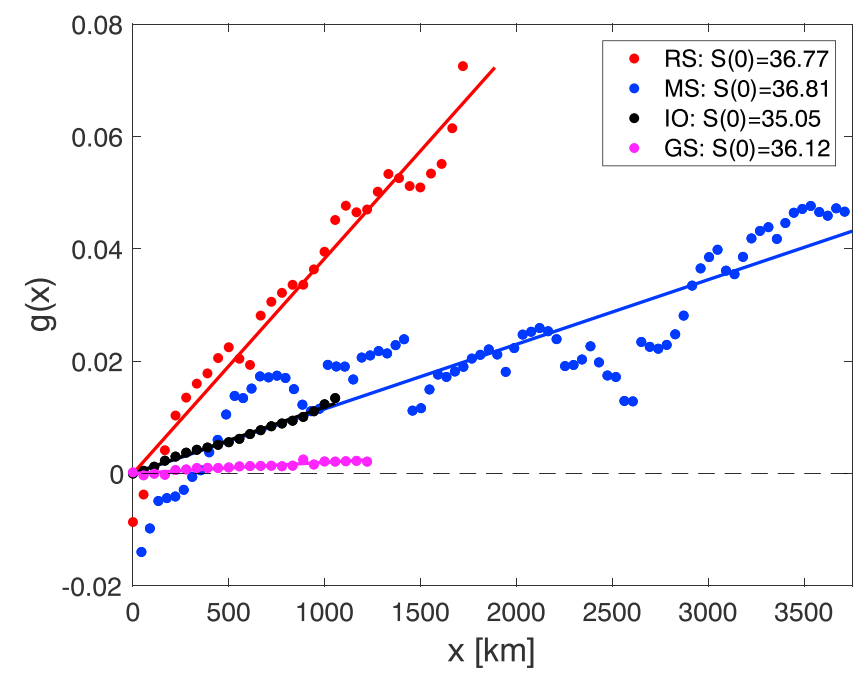

Figure 4. The calculated $\Delta S(x) / S(0)$ functions and the corresponding $g(x)$ linear regression lines in the four transects described in section 3.1.1. Note that the black and blue $\mathrm{g}(\mathrm{x})$ lines are indistinguishable from one another. RS $=$ Red Sea; $\mathrm{MS}=$ Mediterranean Sea; $\mathrm{IO}=$ Indian Ocean; GS = Gulf Stream. of the zonal transect that is derived by averaging data from $O\left(20^{\circ}\right.$ latitude $)$ and to fresh water outflows from the Adriatic and Aegean Seas. Indeed, $R^{2}$ is higher (0.83) in the first (i.e., western) $1,400 \mathrm{~km}$ of the transect, where no major fresh water sources exist. Clearly, the largest total salinity difference: $\Delta S_{\max }=S\left(x_{\max }\right)-\mathrm{S}(0)$ is encountered in the hot, dry, and slowly flowing RS, while the lowest total salinity difference is in the fast flowing GS.

Having determined the intercepts of the linear regression lines that best fit $S(x)$ in the four SODA transects, we now proceed to determine the $g(x)$ functions in each transect, equation (7), that yield the evaporation length. The four $\Delta S(x) / S(0)$ functions along with their best fitting $g(x)$ regression lines are shown in Figure 4, and they clearly demonstrate that the slope of $g(x)$ regression line is largest in the hot and dry RS, smallest in the fastmoving GS, and has intermediate values in the MS and IO. As in Figure 3 the zonal variability in the MS causes the large undulations in the observed $\Delta S(x) / S(0)$ about the best fitting $g(x)$.

The variations of salinity and stable isotopes observed in the two cruises along the RS and IO transects (Figure 5) are similar to those in the SODA salinity transects. Two points should be underlined: The first is that the $R^{2}$ values of the linear regression lines are as high as those in SODA 

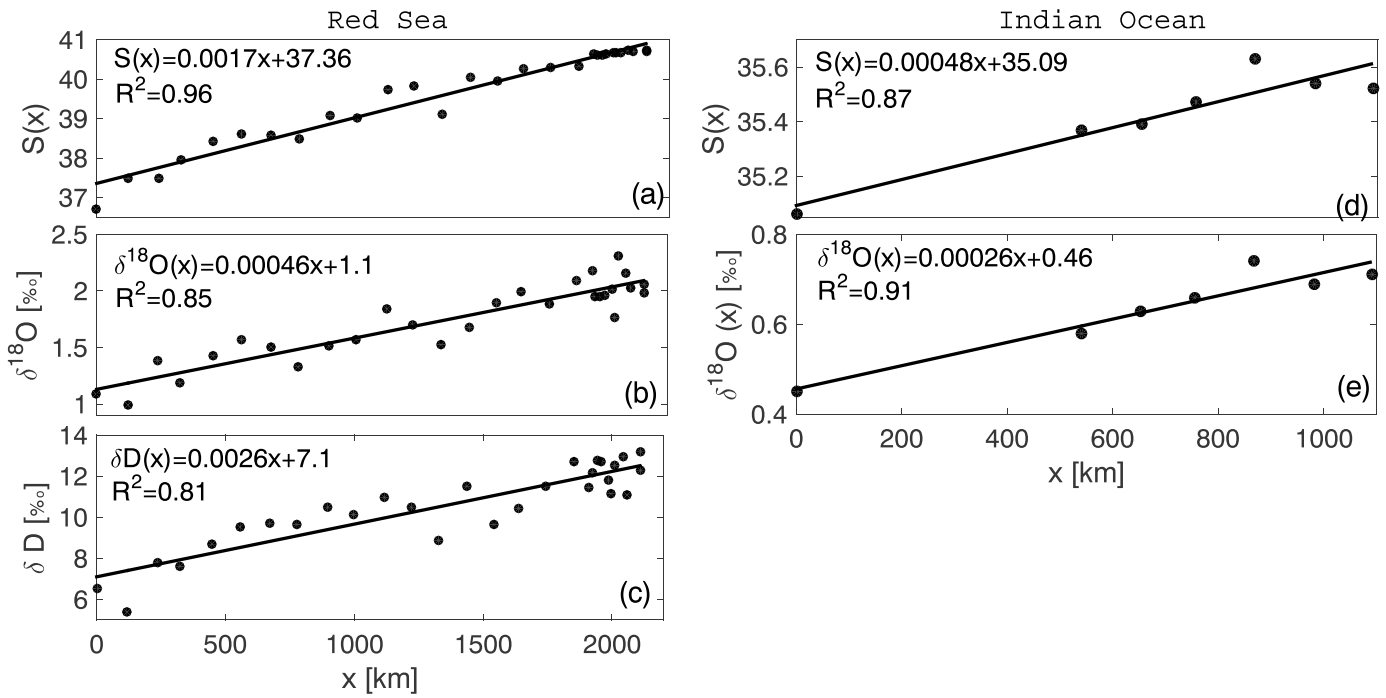

Figure 5. The calculated $g(x)$ functions from salinity and stable isotope data taken in the cruises along the RS and IO transects described in section 3.1 .2 .

even though the cruise data are snapshots of order 1-2 weeks and not multiyear means. The second is that $R^{2}$ values of the stable isotopes cannot be distinguished from those of salinity.

A summary of the values of the evaporation lengths, $L$, estimated from the calculated $g(x)$ regression lines in the way described in section 3.2 for all transects described in section 3.1 are shown in Table 1. In the RS the estimates of $L$ based on stable isotopes variations agree with those of salinity (both SODA and cruise data) but in the IO the $\delta^{18} O$ data yields a value of $L$ that is about half that derived from salinity data (again-both SODA and cruise data, indicating that the $\delta^{18} O$ data underestimate $L$ ). Clearly, the evaporation length attains its largest value in the fast moving GS as anticipated from the dependence of $L$ on $u$ in equation (3) and its lowest value is encountered in the hot and dry RS where $q$ is large.

\section{Discussion}

Direct methods for estimating net evaporation rates in a basin require that all components of the heat budget be determined, including meteorologic variables (e.g., long-wave and short-wave radiation, winds, airtemperature, relative humidity) and oceanographic variables (e.g., sea surface temperature, heat content throughout the water column, mixed-layer depth). However, values of meteorologic variables that are required for the implementation of the bulk formulae are seldom available with sufficient accuracy, frequency, and spatial homogeneity (e.g., relative humidity or half-hourly wind speeds, see WGASF Group, 2000). The newly proposed evaporation length $L=u h(0) / q$ can be used for constraining the estimates of $u, q$, and $h(0)$, based on oceanographic transects of either salinity or isotopic composition.

The values of the evaporation length calculated in the four transects studied here show that this new variable reaches its maximal value of $5 \cdot 10^{5} \mathrm{~km}$ in the GS and its minimal value of $25 \cdot 10^{3} \mathrm{~km}$ in RS. This factor of 20 difference in the values of $L$ in the two basins can be easily explained by the huge gap in the propagation speeds, $u$, which according to SODA surface velocity are $O(0.02 \mathrm{~m} / \mathrm{s})$ and $O(0.7 \mathrm{~m} / \mathrm{s})$ in the RS and GS, respectively. On the other hand, the values of $L$ in the MS and the IO are quite similar which might imply that $u, q$, and $h(0)$ have similar values in the two basins. The calculated low values of $\mathrm{L}$ in the RS are consistent with previous estimates of high evaporation rates there (Ben-Sasson et al., 2009; Berman et al., 2003; Sofianos et al., 2002).

A surprising finding of the calculations presented above is the agreement between estimates of $L$ that arise from (the routinely measured) salinity and those obtained from changes in stable isotope compositions along the transect. In the RS both hydrogen and oxygen isotopes, $\delta D$ and $\delta^{18} O$, yield values of $L$ that are very similar to that derived from both SODA salinity transect and cruise (i.e., snapshot) data. For some unknown reasons the estimate of $L$ derived from salinity observation aboard the cruise $\left(84 \cdot 10^{3} \mathrm{~km}\right)$ varies only 
slightly from that derived from the SODA salinity transect $\left(74 \cdot 10^{3} \mathrm{~km}\right)$ but the value of $L$ derived from $\delta^{18} \mathrm{O}$ is merely $35 \cdot 10^{3} \mathrm{~km}$.

The increase in the isotopic data collected globally in the ocean offers an independent confirmation of estimating $L$ that can be routinely derived from the simpler to use salinity data. The excellent linear fit of the least squares lines of any of the observed variables in all basins makes our model relevant to any study of evaporation in the world ocean. The independent constraint proposed here is currently the only practical check on the elaborate and complicated method of detailed heat budget calculation.

The physical interpretation of the model parameter $h(0)$ and the determination of its value have not been addressed in the present study and are left for future studies. Possible interpretations of $h(0)$, which is introduced here as the depth to which evaporation affects the properties of the water, are as follows: the depth of the seasonal mixed layer, the depth of the turbulent surface layer, the depth of the downward wind mixing, etc. It is also possible that it is unrelated to any previously known surface layer and represents a new depth scale. According to equation (3), $h(0) u=q L$, so the values of $L$ in Table 1 should be multiplied by $q$ (that varies only slightly between the four transects, say $q \approx 1.8 \mathrm{~m} /$ year) in order to estimate $h(0) u$. Thus, the ratio between the values of $L$ in Table 1 is also the ratio between $h(0) u$ in the four transects. An estimation of $h(0)$ requires that salinity or stable isotopes be quantified to derive $L$ followed by an estimate of $u$ throughout the entire layer thickness, $h(0)$, instead of the surface values only. However, this averaging requires that the value of $h(0)$ be known prior to the evaluation of $L$ so it can only be done in a complicate iterative procedure.

\section{Acknowledgments}

The salinity and $\delta^{18} O$ data collected aboard the Indian Ocean cruise described in Srivastava et al. (2007) can be accessed at this website (https:// www.nodc.noaa.gov). The salinity, $\delta^{18} O$ and $\delta D$ data collected during the Red Sea cruise of the Interuniversity Institute for Marine Sciences, Eilat, described in Steiner et al. (2014) and can be accessed in the supporting information section of doi: 10.1073/ pnas.1414323111. H. B. acknowledges the support provided by the Eshkol Foundation of the Israel Ministry of Science.

\section{References}

Ali, E. B., Churchill, J. H., Barthel, K., Skjelvan, I., Omar, A. M., de Lange, T. E., \& Eltaib, E. (2018). Seasonal variation of hydrographic parameters off the Sudanese coast of the Red Sea. 2009-2015. Regional Studies in Marine Science, 18, 1-10. https://doi.org/10.1016/j. rsma.2017.12.004

Appelo, C. A. J., Postma, D. (2005). Geochemistry, groundwater and pollution (Chap. 2, pp. 33-37). London: CRC Press.

Ben-Sasson, M., Brenner, S., \& Paldor, N. (2009). Estimating air-sea heat fluxes in semienclosed basins: The case of the Gulf of Elat (Aqaba). Journal of Physical Oceanography, 39(1), 185-202. https://doi.org/10.1175/2008JPO3858.1

Berman, T., Paldor, N., \& Brenner, S. (2003). Annual SST cycle in the eastern Mediterranean, Red Sea and Gulf of Elat. Geophysical Research Letters, 30(5), 1261. https://doi.org/10.1029/2002GL015860

Carton, J. A., Chepurin, G. A., \& Chen, L. (2018). SODA3: A new ocean climate reanalysis. Journal of Climate, 31(17), 6967-6983. https:// doi.org/10.1175/JCLI-D-18-0149.1

Cayan, D. R. (1992). Variability of latent and sensible heat fluxes estimated using bulk formulae. Atmosphere-Ocean, 30(1), 1-42. https:// doi.org/10.1080/07055900.1992.9649429

Clark, I. D., \& Fritz, P. (1997). Environmental isotopes in hydrogeology (p. 308). London: CRC Press.

Craig, H., Boato, G., \& White, D. E. (1956). Isotope geochemistry of thermal waters. In Proc. 2nd conference on nuclear processes in geologic settings (Vol. 19, pp. 19-44). Washington, DC: National Academy of Science, National Research Council.

Dansgaard, W. (1964). Stable isotopes in precipitation. Tellus A, 16(4), 436-468. https://doi.org/10.3402/tellusa.v16i4.8993

Knauss, J. A. (1978). Introduction to physical oceanography. Englewood Cliffs, NJ: Prentice Hall.

Kondo, J. (1975). Air-sea transfer coefficients in diabatic conditions. Boundary-Layer Meteorology, 9(1), 91-112. https://doi.org/10.1007/ BF00232256

Majoube, M. (1971). Fractionnement en oxygene-18 et en deuterium entre leau et sa vapeur. Journal de Chimie Physique, 68(7-8), 1423-1436. https://doi.org/10.1051/jcp/1971681423

Manabe, S., \& Smagorinsky, J. (1967). Simulated climatology of a general circulation model with a hydrologic cycle. II-Analysis of the tropical atmosphere. Monthly Weather Review, 95(4), 155-169. https://doi.org/10.1175/1520-0493(1967)095<0155:SCOAGC>2.3.CO;2

POEM Group (1992). General circulation of the eastern Mediterranean. Earth Science Reviews, 32(4), 285-309. https://doi.org/10.1016/ 0012-8252(92)90002-B

Sofianos, S., Johns, W., \& Murray, S. (2002). Heat and freshwater budgets in the Red Sea from direct observations at Bab el Mandeb. Deep Sea Research Part II: Topical Studies in Oceanography, 49(7), 1323-1340. https://doi.org/10.1016/S0967-0645(01)00164-3

Srivastava, R., Ramesh, R., Prakash, S., Anilkumar, N., \& Sudhakar, M. (2007). Oxygen isotope and salinity variations in the Indian sector of the Southern Ocean. Geophysical Research Letters, 34, L24603. https://doi.org/10.1029/2007GL031790

Steiner, Z., Erez, J., Shemesh, A., Yam, R., Katz, A., \& Lazar, B. (2014). Basin-scale estimates of pelagic and coral reef calcification in the red sea and western Indian Ocean. Proceedings of the National Academy of Sciences, 111(46), 16,303-16,308. https://doi.org/10.1073/ pnas.1414323111

Talley, L. D., Pickard, G. L., Emery, W. J., \& Swift, J. H. (2011). Descriptive physical oceanography: An introduction (6th ed.). London, UK: Academic Press. https://doi.org/10.1016/B978-0-7506-4552-2.10001-0

WGASF Group (2000). Intercomparison and validation of ocean-atmosphere energy flux fields. Final Report, Joint WCRP/SCOR Working Group on Air-Sea Fluxes, WMO, Geneva, Switzerland. 